\title{
A Comparison of different measurement technologies for plant's height and diameter determination
}

\author{
Ramandeep Kaur \\ Lecturer in ECE (Shaheed Bhagat Singh State Technical Campus, Ferozepur, India)
}

\begin{abstract}
The measurement of a plant growth without interrupting its natural growth is essential since its diameter and height are related to the development of a tree as well as the water contents. Many studies have found that the variation of the diameter and height of plant stem has a prominent correlation ship with water status, temperature, light and humidity. Evaluation of these aspects by variation of plant stem is suitable for automatic monitoring in a long period of time. Thus due to foresaid factors automatic irrigation approach is of utmost importance these days. A comparison of different measurement methods used for the discovery of plant's parameters like height and diameter has been discussed in detail in this paper. The advantages and disadvantages of these technologies are comprehensively dealt in this study.
\end{abstract}

Keywords : Edges, growth, stem diameter and height, strain gauge

\section{Introduction}

Process of plant expansion will be influenced by environmental conditions and topography. Currently, there are various techniques for measuring stem diameter and height. Several experiments were conducted to investigate the development and expansion of plant. The results of these experiments indicate that the variation of the diameter of plant stem, height, temperature and humidity has a prominent correlation ship with the water status. Since the 1980s, some researchers use the variations of the growth of plant as the indicators of the water status for irrigation. Evaluating the water status by variation of plant growth is suitable for automatic monitoring in a long period of time without destruction of the tissue of plant.

There is need to measure dynamics of plant to understand how plants respond to variation in water content and long term water conditions. Many studies have found that variation of the diameter and height of plant stem has a prominent correlation ship with water status, temperature, light and humidity. Evaluating these aspects by variation of plant stem is suitable for automatic monitoring in a long period of time. Thus due to foresaid factors automatic irrigation approach is of utmost importance these days.

At the present times computer vision is being developed from the notion of not only directly influencing its own field, rather to aid and influence other environments like automatic control robotics, neurobiology, artificial intelligence, machine learning and image processing. The body of knowledge generated in computer vision is making an impact beyond its horizon applications. As a scientific discipline, computer vision is concerned with the theory for building artificial systems that obtain information from images. As a technological discipline, computer vision seeks to apply the theory and models of computer vision to the construction of computer vision systems. Sub-domains of computer vision include scene reconstruction, incident detection, tracking, object recognition, learning, indexing, motion estimation, and image restoration. Among the various attributes of computer vision discussed above the present work is focused on measurement of height and diameter of plant using efficient measurement technology.

\subsection{Measurement using tapes and calipers}

\section{Measurement Technologies}

These are the most common and traditional instruments for measuring the growth of a plant. The tape is wrapped around the tree to measure circumference. The tape should be held relatively firmly (but avoid stretching). The tape should also be wrapped around the bole in a perpendicular plane to the stem axis. Keeping the tape numbers right side up (as in the photograph) reduces the chances of incorrectly reading the scale - when upside down errors like x.4 being recorded as x.6 are common. Some diameter tapes are 'rounded down' to the nearest $\mathrm{cm}$ class, others (used more for research purposes) can read to the nearest $\mathrm{mm}$. Dbh tapes are either plastic-coated fiberglass or steel: the former can stretch as they wear and thus should be replaced as necessary. The steel tapes are expensive and not very hard- wearing.

Calipers are also very common and offer a quick way of measuring tree dbh. They are made of steel or alloy. Calipers are comprised of a fixed arm, scale and moveable arm. The fixed arm is placed along one side of the tree at the desired height. The moveable arm is then placed flush against the other side of the tree and the scale is read directly. The calipers must be located perpendicular to the stem axis. The same rules for dbh tapes apply but with calipers, two measurements should be made on each tree at right angles to each other and the 
average recorded. The most common fault with calipers is excessive movement in the mobile arm and thus they must be regularly checked to ensure that the two arms are parallel.
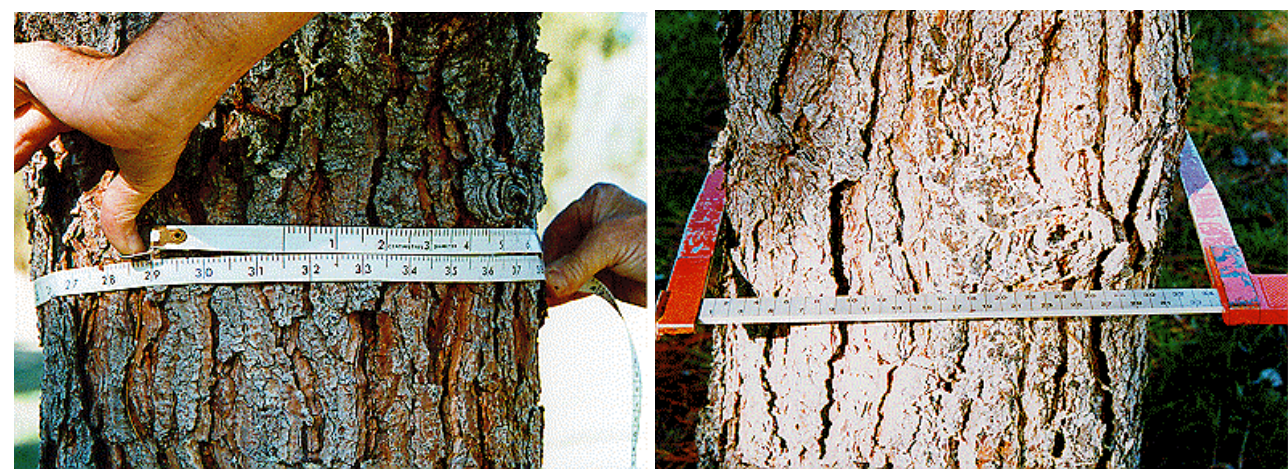

Figure 2.1 (a) Tape , (b) Caliper

\subsection{Strain Gauge Measurement Technology}

More recent designs use strain gauges to detect changes in stem size through deformations in an attached metal housing. Ensor described here is light, can remain attached to stems for months to years, and can detect micron-size changes. This sensor can measure diameter changes for long periods of time as long as stem diameter growth is slow as in desert shrubs. The sensor, in a stiff metal housing, has to be reset to a new zero point if the diameter increases by more than $1 \mathrm{~mm}$ to avoid deforming the stem or breaking the device.

The dendrometer is constructed out of two aluminum bars attached to a flexible stainless steel band on which the strain gauges are attached. The flexible stainless steel band is $25.4 \mathrm{~mm}$ wide and $0.76 \mathrm{~mm}$ thick. The sensor is attached by screwing a bolt with a curved anvil at its end through one of the aluminum bars pressing the stem or fruit between two anvils (Fig 2.1). Four strain gauges are connected in a full Wheatstone bridge arrangement and attached in the middle of the flexible band with two on each side. The DEX70 dendrometer showing the aluminum bars and stainless steel band with attached strain gauges clamped on to an apple fruit and the larger, DEX100 dendrometer clamped on to a tree trunk shown in fig 2.2.

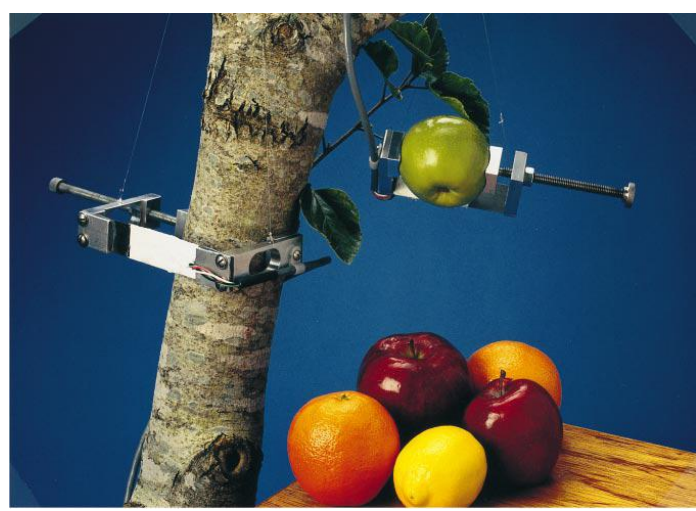

Figure 2.2 DEX70 dendrometer

Dimensions of the device are given in Fig 2.3.

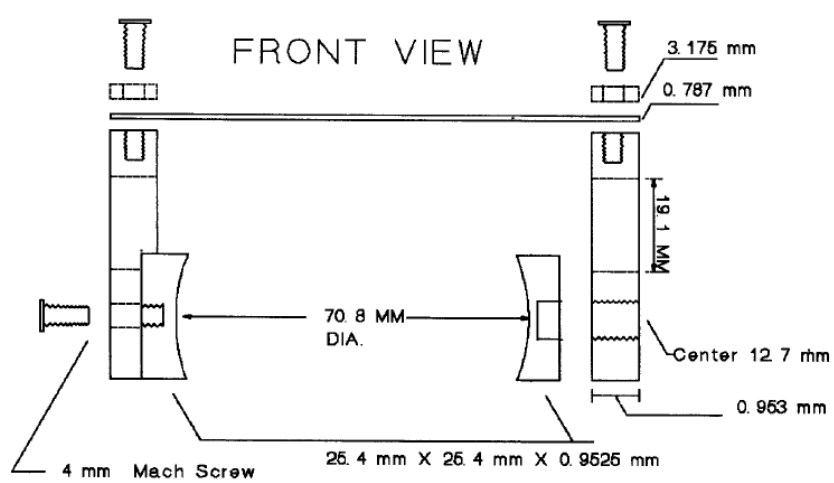

Figure 2.3 Dimensions of the metallic pieces of the DEX70 dendrometer 
Fine-wire copper-constantan thermocouples were apprised to the metal housing of each dendrometer to record sensor temperature. Air temperature was measured with fine-wire thermocouples at the same height as the apple with the dendrometer attached. As the stem or fruit diameter changes, differences in the relative tension experienced by the strain gauges are measured as a millivolt signal. Dendrometer and thermocouples were attached to a data logger (CR7X, Campbell Scientific, Logan, UT) with data averaged over $1 \mathrm{~h}$ intervals for the first few days of observation and over $10 \mathrm{~min}$ intervals for the remainder. Millivolt signals are converted to millimeter units after calibration. This data logger has a resolution of $0.166 \mathrm{mV}$ over a full scale range of \pm 5 $\mathrm{mV}$. Calibration was done on each dendrometer by altering the spacing of an internal micrometer and recording the millivolt signal. Diameters ranged from 0 to $12.7 \mathrm{~mm} \mathrm{[2].}$

\section{Disadvantages}

1. It is required to change sensors of different scale to fit the different range of diameters.

2. It only provides increment and decrement of the diameter while leaving the real value of the stem diameter unknown.

3. The sensor also interrupts the natural growth of the plants.

\subsection{LVDT Measurement Technology}

Linear variable displacement transducers (LVDTs) can continuously and accurately monitor stem diameter. Recent improvements in LVDT robustness, data acquisition systems and computer controlled irrigation make using this instrument more feasible in irrigation management.

The LVDT, used to measure stem diameter, was attached to the plant with a special holder (Figure 2.4) which allowed accurate measurement of both diurnal changes and long term growth. All parts are of nonmagnetic stainless steel except for the nonmagnetic beryllium-copper spring and the glass rod with low thermal expansion. The plant is tied to the holder with soft rubber tubing to minimize movement in the wind. The spring keeps the front stem positioned pressed against the stem so that the core will retreat if the stem shrinks. To minimize hysteresis when measuring soft tissues, springs with small spring constants are used.

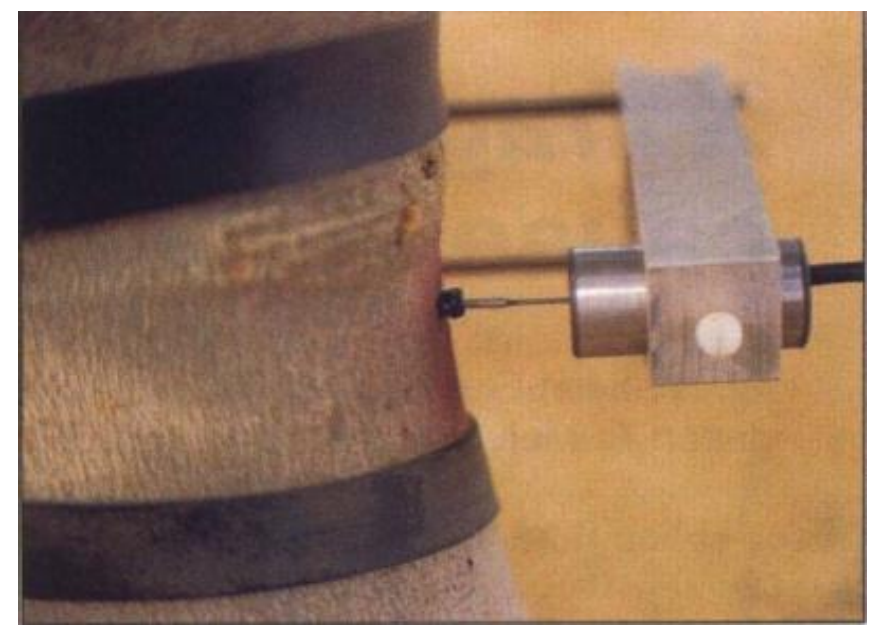

Figure 2.4 Linear variable displacement transducer (LVDT)

As the stem grows, the micrometer is turned to recenter the core and the amount of growth is recorded. Thus, the instrument has both a high level of precision and a large range. The output from the LVDT is amplified and recorded on a strip-chart recorder. Calibration can be done in situ by merely moving the micrometer head a known distance and recording the change in output [3].

\section{Disadvantages}

1. Strain gauges are relying on the change in gauge resistance in determining the strain within the gauge section.

2. Measuring the growth of plant using strain gauge does not provide accurate values of plant's growth. It required large precision.

3. The output signal is weak and unwanted signal and interference is also introduced. Thermal effect also arises in strain gauge method. 


\subsection{PC Based Image Processing System}

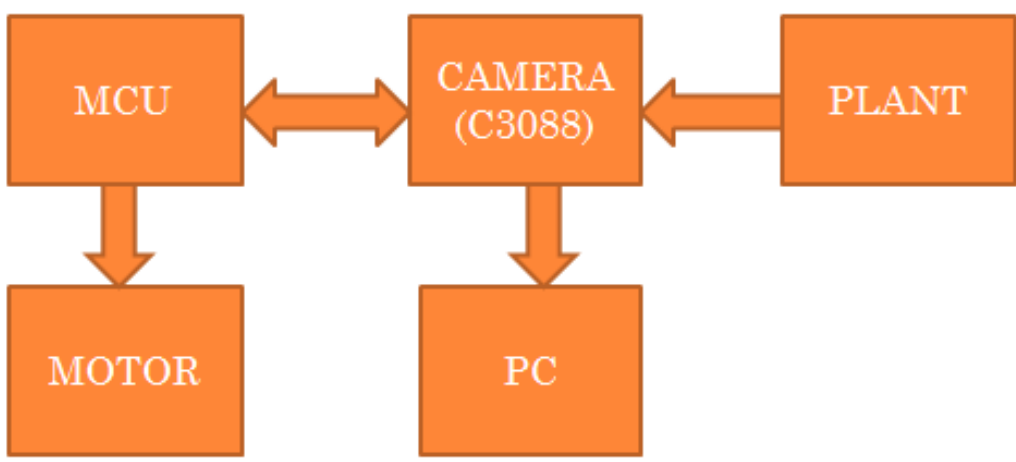

Figure 2.5 Block Diagram of System

Fig 2.5 shows the general configuration of the system. The basic structure consists of digicam module, controller and PC. The system comes into an action when a user presses the button, mounted on it for the user interface. In first module, there is camera interfaced to AVR controller to acquire an image. Main advantage of this camera is that it has digital output. Digital port that supplies a continuous 8-16 bit wide range data stream. It will capture the image of plant at different interval of time. Digicam pass the data to the PC through USB. All the functions of camera contrast, brightness, intensity etc. are programmable through $\mathrm{I}^{2} \mathrm{C}$ interface. For further processing of the image, it is fed to the PC. In this module embedded programs with the functions of image acquisition, edge detection (Sobel Edge Detection) and data storage have been developed in MATLAB. Such programs enable us to automatically measure and record the various parameters of a plant. PC sends commands to camera through controller using RS232 serial communication standard. Motor is used to move the camera on belt to capture images of different plants.

A MATLAB GUI has been developed for performing Sobel Edge Detection on a plant image to determine its height, maximum and minimum width. Fig 2.6 is presenting the plant image with highlighted edges as well as the measured values of height, maximum and minimum width of a plant in terms of pixels [4].

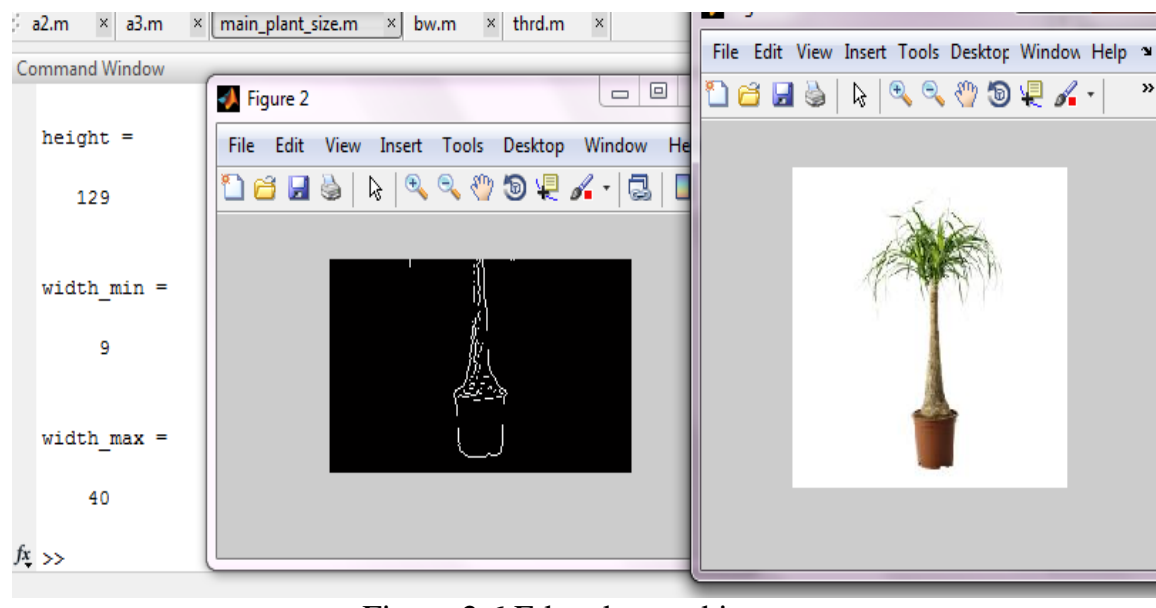

\section{Disadvantages}

Figure 2.6 Edge detected image

1. In this kind of system the camera need to transfer the image to PC. Because the bandwidth of the interface connected to the camera and PC is limited, the image usually needs compression and loses some accuracy in the vision measurement system. If we do not use the compressed communication method, the cost of the system will increase significantly.

2. The image processing PC itself is space consuming and not easy to move. The measurement system needs to work in the environment of high humidity, the risk of wetted by irrigation even the interruption of insect. If we place image processing PC in place like this, we need to set specific cabinet contenting the measurement system. This is inconvenient for the experimentation.

\subsection{Measurement Using Smart Camera and Embedded System}

It is a compact, mobile measurement system choosing DSP embedded Smart Camera VC-4472 as the main components of its device. The smart VC-4472 has integrated three TMS320C64xx DSP cores and has a higher image processing power than the conventional PC-based image processing system. The real-time 
processing of the image can be accomplished on the camera alone without using PC. Secondly, the imaging system of the device uses a background illumination source to improve the quality of images of stems.

Smart cameras with the function of image acquisition, image processing, communication and data storage are chosen to the core component of the system to prominently reduce the size of our vision measurement system. The DSP in the smart camera has computational power of 8000 MIPS rivaling a $7.2 \mathrm{GHz}$ Pentium which is far more than the industrial PC. When the original image with the size of $1600 * 1200$ is read into the DRAM of the camera, parameters measuring programs read the image directly from the DRAM without compression or transfer to calculate the parameters we need and store them in to the flash disk in the smart camera. In this case, we do not need to transfer or compress the original image. As a result, this method reduces the load of the communication and increases the accuracy of the parameter. (Compression or transfer image through analog interface will reduce its quality.) In order to eliminate the interference of the sunlight variation, our system integrates the independent light source. The structure of the vision measurement system is show in fig $2.7[5]$.

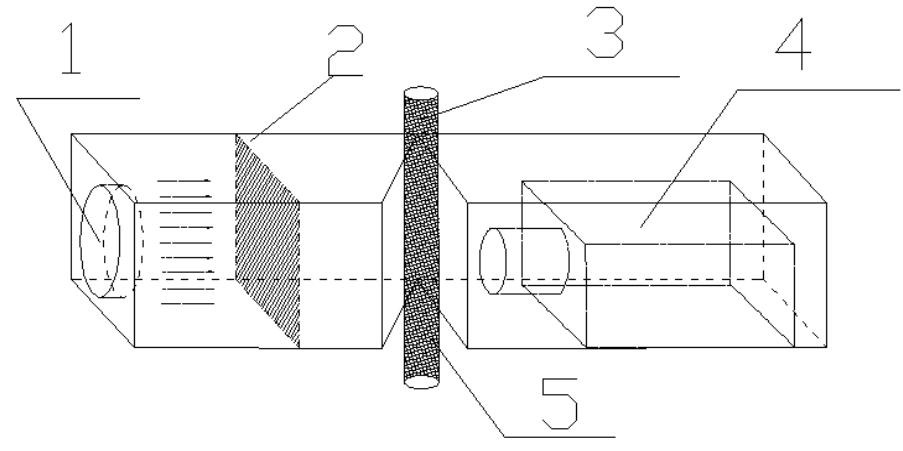

Figure 2.7 The construction of the vision measurement system

\section{Conclusion}

From the above discussion and comparison it is concluded that the measurement using smart camera is more efficient than traditional methods. In this method measuring device is simple and compact. Edges are smoother in comparison to PC based image processing system. PC based system does not provide accurate and précised values. Strain gauge method provides only increment and decrement of diameter, while leaving the real value of the stem diameter unknown. Both the strain gauge and LVDT technologies interrupt the natural growth of plants. The main advantage of smart camera technology is that it will not interrupt the growth of plant which is our prime objective.

\section{References}

[1] Ariff, E.A.R.E. Suratman, M.N.Abdullah," Stomatal conductance, chlorophyll content, diameter and heightin different growth stages of rubber tree (Hevea brasiliensis) saplings," Business, Engineering and Industrial Applications (ISBEIA), 2011 IEEE Symposium, pp.84-88, 25-28 Sept. 2011.

[2] S.O.Link, M.E.Thiede and M.G.van Bavel, "An improved strain-gauge device for continuous field measurement of stem and fruit diameter,” Journal of Experimental Botony, Vol.49, No. 326, pp. 1583- 1587. September 1998.

[3] Elias Fereres, David Goldhamer et.al., "Continuous trunk diameter recording can reveal water stress in peach trees," California Agriculture, Vol. 53, No. 4, July-August 1999.

[4] Ramandeep Kaur and Balwinder Singh, "Design and development of sapling Monitoring System", International Journal of Computer Science, Engineering and Application(IJCSEA) Vol. 3, No. 5, October2013.

[5] Jiaxing Che, Chunjiang Zhao, Yunhe Zhang, Cheng Wang, Xiaojun Qiao, Xinlu Zhang, "Plant stem diameter measuring device based on computer vision and embedded system," World Automation Congress (WAC), 2010, pp.51-55, 19-23 Sept. 2010.

[6] N.M.Khairi, M.A.Marni Shah Rizam, M.S.B. Tahir, N.M. Naimah, M.I. Zainol Abidin,"Optimization of strain gauge for stem measurement using PIC based instrumentation," System Engineering and Technology (ICSET), 2011 IEEE International Conference, pp.196-199, 27-28 June 2011.

[7] Klepper B, Browning VD, Taylor HM. "Stem diameter in relation to plant water status", PlantPhysiology,1971(48) 683-685.

[8] Mcburney T, Costigan PA. "The relationship between stem diameter and water potentials in stems of young cabbage plants", Journal of Experimental Botany, 1984(35)1787-1793.

[9] C.Y. Liao, V.V. Podrazsky, and G. B. Liu, "Diameter and height growth analysis for individual white pine trees in the area of Kostelec nad Cernymilesy," Journal of Forest Science, vol. 49, pp. 544-551, 2003.

[10] T. Kärki, M. Maltamo, and K. Eerikäinen, "Diameter distribution, stem volume and stem quality models for grey alder (Alnus incana) in eastern Finland," New Forests, vol. 20, pp. 65-86, 2000.

[11] X.H. Sang, Y.Y. Wu, Y.G. Zhao, and M.Y. Xu, "Comparison of growth of four types of Atractylodes Lancea (Thunb.) DC," Journal of Anhui Agricultural Sciences, vol. 36, pp. 7726 - 7727, 2008. 